\title{
Die Quantenempfindlichkeit der Uranyloxalatphotolyse
}

\section{Doctoral Thesis}

Author(s):

Büchi, Paul Felix

Publication date:

1924

Permanent link:

https://doi.org/10.3929/ethz-a-000099659

Rights / license:

In Copyright - Non-Commercial Use Permitted

Originally published in:

Sammelband ETH-BIB 


\title{
Die Quantenempfindlichkeit der Uranyloxalatphotolyse.
}

\author{
ஊ \\ VON DER \\ EIDGENÖSSISCHEN TECHNISCHEN \\ HOCHSCHULE IN ZÜRICH \\ ZUR ERLANGUNO \\ DFR WÜRDE EINES DOKTORS DER \\ TECHNISCHEN WISSENSCHAFTEN \\ GENEHMIGTE \\ PROMOTIONSARBEIT \\ VORGELEGT VON \\ PAUL F. B ÜCH I \\ DIPL. INGENIEUR-CHEMIKER \\ AUS OBERWINTERTHUR.
}

Referent: Herr Prof. Dr. E. Baur.

Korreferent: Herr Prof. Dr. W. D. Treadwell

372

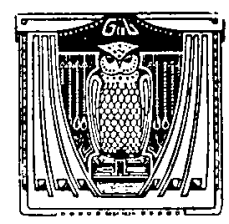

Leipzig

Druck von Breitkopf \& Haertel

1924. 
steinschen Theorie zur Folge hatten, also neben die von W. Noddak') and H. Grüss') untersuchte Lichtreaktion des Chlors auf Trichlorbrommethan, und des Sauerstoffs auf denselben Akzeptor 2).

Der Zerfall des Uranyloxalates im sichtbaren Licht zeigt also die einfachsten Gesetzmässigkeiten, welche von einem photochemischen Prozess erwartet werden können:

Reaktion nullter Ordnung ${ }^{3}$ ); weitgehende Unabhängigkeit von Zusätzen, sofern letztere nicht den Oxalatkomplex zerstören; Temperaturkoeffizient praktisch gleich 1 (Bacon; Bruner und Kozak); auf ein absorbiertes Lichtquantum wird ein Molekül Oxalsäurc zersetzt (Einsteinsches Gesetz).

\section{Zusammenfassung.}

1. Die Photolyse der Systeme von Uransalzen mit Oxalsäure ist ein Lichtzerfall des undissoziierten Uranyloxalats $\left[\mathrm{UO}_{2} \mathrm{C}_{2} \mathrm{O}_{4}\right]$ bzw. des Ions $\left[\mathrm{UO}_{2}\left(\mathrm{C}_{2} \mathrm{O}_{4}\right)_{2}\right]^{\prime \prime}$.

2. Die Zersetzungsgeschwindigkeit ist, sofern die Oxalsäure in überäquimolarer Menge gegenüber dem Uransalz vorhanden ist, unabhängig von der 0xalsäurekonzentration und zeitlich konstant. Für unteräquimolare Mengen von Oxalsäure ergibt sich eine Lichtempfindlichkeit, welche ungefähr der Konzentration der Oxalsäure proportional geht (Knickpunkt bei äquimolarem Gemisch).

3. Die relativ grosse Beständigkeit des Komplexes erklärt:

a) Die quantitative Gleichheit der Zersetzungsgeschwindigkeit für Systeme von Uranylsulfat, -nitrat und -oxalat mit Oxalsäure.

b) Die Unabhängigkeit der Photolyse von Zusätzen - nämlich $\mathrm{O}_{2}, \mathrm{HCl}, \mathrm{H}_{2} \mathrm{SO}_{4}, \mathrm{H}_{2} \mathrm{CO}_{2}$ - sofern diese nicht den Komplex zerstören.

4. Die Acidität der Lösung ist bestimmend für das Ausmass der Ameisensäurebildung (Antibasie) und der Bildung von Uranosalz (Symbasie).

5. Der Bruttovorgang, die Zersetzung der Oxalsäure in wässeriger Lösung, ist ein affinitätsgemäss freiwilliger.

1) Zeitschr. f. Elektrochemie 27, 359 (1921).

2) Zeitschr. f. Elektrochemie 29, 144 (1923).

3) Die hier gefundene Abhängigkeit der Lichtempfindlichkeit von der Konzentration der Oxalsäure, wenn diese in unteräquimolarer Menge vorhanden ist, hat ihre Erklärung durch das optische Verhalten der Lösung gefunden, ohne dass die Photolyse chemisch in ihrer Art geändert wird. 
314 Paul F. Büchi, Die Quantenempfindlichkeit der Uranyloxalatphotolyse.

6. Auf ein absorbiertes Lichtquantum wird e in Molekül Oxalsäure zersetzt, gemäss dem Einsteinschen Gesetz.

7. Für die Uranylformiatzersetzung in schwefelsaurer Lösung gilt für das Anfangsstadium eine Quantenempfindlichkeit von $0.7 \pm 0.2$, d. h. es werden auf ein absorbiertes Quantum etwa 0.7 Moleküle Uranosalz gebildet, bzw. ebensoviel Moleküle Ameisensäure umgesetzt.

Diese Arbeit wurde im physikalischen Laboratorium der Eidg. Techn. Hochschule von Zürich ausgeführt. Meinem hochverehrten Lehrer Herrn Prof. Dr. E. Baur möchte ich hier für sein stets förderndes Interesse an meiner Arbeit danken. 